\title{
PERCEPÇÃO MATERNA DA DESIDRATAÇÃO EM CRIANÇAS COM DIARRÉIA. ESTUDO DE CONCORDÂNCIA COM DIAGNÓSTICO MÉDICO*
}

\author{
Maria do Carmo Leal** \\ Rosana Iozzi da Silva*** \\ Silvana Granado Nogueira da Gama**
}

\begin{abstract}
LEAL, M. do C. et al. Percepção materna da desidratação em crianças com diarréia. Estudo de concordância com diagnóstico médico. Rev. Saúde públ., S. Paulo, 24: 196-203, 1990.

RESUMO: Realizou-se estudo em um hospital infantil do Rio de Janeiro, em crianças hospitalizadas por diarréia, no período de janeiro $/ 87$ a fevereiro/88, com o objetivo de destacar a percepçāo materna dos sinais e sintomas da desidrataçảo em crianças menores de três anos de idade, confrontando-a com o diagnóstico médico. Os sinais e sintomas mais identificados pelas mães foram o número de evacuações, número de vômitos, estado da sede e dos olhos. As mães tiveram dificuldade em perceber a quantidade de urina, o estado de umidade da boca e língua e turgor da pele. Identificaram estes sinais quase sempre como normais ou no máximo, moder adamente alterados. As mães que tenderam a subestimar a gravidade da desidratação apontada pelo médico têm nível de escolaridade baixo, filhos desnutridos e maior dificuldade de acesso ao hospital. Já as que tenderam a superestimar a gravidade, têm melhor nível de instrução, filhos melhor nutridos, maior facilidade de acesso ao hospital, bem como passaram por um menor número de serviços de saúde antes de chegar ao hospital estudado. As mães que concordaram com o diagnóstico médico classificaram-se em uma situação intermediária, embora se aproximando mais das que subestimaram a gravidade. Aquelas mães que já haviam vivenciado um episódio de desidratação anterior no seu filho não apresentaram maior concordância com o diagnóstico do médico, mostrando que o repasse de informação no serviço de saúde foi nulo ou inadequado.
\end{abstract}

DESCRITORES: Mães. Percepção. Desidratação, epidemiologia.

\section{INTRODUÇÃO}

As percepçóes expressas pela população a respeito da saúde e da doença envolvem formas particulares de ouvir e olhar seu próprio corpo, as quais são freqüentemente desconsideradas dentro da relação entre médicos e pacientes ${ }^{4}$.

A assistência médica obedece a modelos de conhecimento e prática que conferem ao profissional da área de saúde, em especial ao médico, a autoridade e responsabilidade absoluta de interpretação dos sinais e sintomas emitidos pelos corpos dos indivíduos. Esse modelo perde o paciente na sua integridade, dirccionando seu olhar cada vez mais a partes, órgãos ou sistemas do organismo ${ }^{2,12}$.

Nos serviços de saúde que atendem à população de baixa renda, o desprestígio social deste grupo é reproduzido na relação médico-paciente, não se passando aí uma relação educativa que envolva comunicação e informação.

A instituição médica o:ganizada dentro de concepções funcionais de maximização da ofcrta de assistência reforça e conforma a atuação do médico ao mero papel de funcionário, voltado mais para indicaciores quantitativos de produtividade do que para a qualidade desta atuação ${ }^{13}$. É neste momento que se teria oportunidade de fornecer informação científica e de educação sanitária, mas a pressa e o autoritarismo que marcam a relação com o paciente levam os médicos, pelo contrário, a cancelar freqüentemente esta oportunidade ${ }^{3}$.

Conhecer e valorizar as percepçōes trazidas pela população implica em ampliar a compreensão, por parte desta população dos valores que possui e que pode conquistar, c não deveria se constituir numa ameaça à autoridade médica, que faz prevalceer o scu saber como único e verdadeiro, supermedicalizando seus procedimentos, normatizando condutas, enfim, limitando a compreonsão da educação à passagem unidirecionada de alguns conhecimentos mais difundidos através de programas dirigidos ${ }^{5}$.

O presente trabalho destaca a percepção materna sobre os sinais e sintomas da desidratação em crianças menores de 3 anos de idade e confronta esta percepção com a classificação médica. Pretendemos apontar neste confronto alguns fatores que

\footnotetext{
* Pesquisa subvencionada pela Financiadora de Estudos e Projetos (FINEP). Processo 43.860.197.00

* Escola Nacional de Saúde Pública - Av. Leopoldo Bulhões, 1480 - $8^{\circ}$ andar - Manguinhos - 21041 - Rio de Janeiro, RJ - Brasil.

* * Bolsista do Concelho Nacional de Desenvolvimento Científico e Tecnológico (CNPq).
} 
contribuem para que a mãe concorde, superestime ou subestime a gravidade da doença, chamando assim a atenção para a valorizaçăo da participação ativa do paciente, enriquecedora da relação que pode se estabelecer entre o profissional de saúde e a populaçăo.

\section{METODOLOGIA}

A realização deste trabalho deu-se no periodo de janeiro de 1987 a fevereiro de 1988, no Hospital Municipal Sales Neto (HMSN). Esta unidade hospitalar de pequeno porte é intensamente procurada pela população para tratamento das doenças entéricas infantis, uma vez que por muitos anos foi a unidade de referência para essas enfermidades.

Foram incluídas 406 crianças na faixa etária de 0 a 3 anos atendidas no setor de emergência e internadas no HMSN, tendo como diagnóstico clínico a doença diarréica. Através da relação de admissão e alta do hospital, foram identificadas todas as crianças internadas. No setor de emergência investigou-se uma amostra das crianças atendidas pelo. fato de a equipe de pesquisadores permanecer apenas três dias por semana naquele hospital. Este grupo era encaminhado a equipe imediatamente após a consulta.

As informaçōes analisadas foram obtidas pelo questionário aplicado por componentes da equipe de investigaçăo treinadas para esta tarefa. Este questionário consistiu de duas partes: uma com informaçð̄es colhidas diretamente do prontuário:

- caracterizaçăo do estado de saúde da criança no momento do atendimento - estado geral, quadro clínico, estado de hidratação, diagnóstico clínico;

- dados antropométricos e sinais vitais peso, altura, tomperatura, freqüência cardíaca e respiratoria;

- conduta médica - medicamentos prescritos, exames solicitados;

e outra com informaçôes obtidas de entrevista com as mães, às quais foi solicitado:

\section{- a identificação da criança;}

- informaçōes rolacionadas ao estado de saúde e intercorrências pregressas à doença atual história gestacional, condiçס̄es de nascimento, doenças anteriores, aleitamento materno;

- informaçס̃es gerais sobre a família e condiçőes sócio-econômicas - moradia, grau de instrução dos pais, condição de saneamento.

A maioria das informaçōes desta parte foi fornecida pela mãe da criança $(87,2 \%)$. Quando não se conseguia obter as informaçoes faltantes diretamente com um familiar das crianças, recuperavam- se alguns dados disponíveis no serviço social do HMSN (12,8\%).

A classificação da hidratação feita pelo médico foi coletada tal como se encontrava registrada no prontuário da criança, que era classificada como normohidratada ou desidratada do Primeiro, Segundo e Terceiro graus. Esta descrição médica não foi por nós validada, nem pelos critérios clínicos, nem pelos de ganho ponderal.

As mães perguntou-se sobre os sinais e sintomas mais comuns da desidrataçăo e, a partir destes dados, foi classificado o estado de hidratação segundo os critérios recomendados pela Organização Mundial da Saúde (OMS) ${ }^{10}$, conforme mostra a Tabela 1.

Os dados desta Tabela permitiram recompor aquilo que era detectado pela mãe como "alterado" e classificar o estado de hidratação em normohidratado, moderadamente e gravemente desidratado, segundo sua percepção.

Uma vez concluída esta "composiçāo" de sinais e sintomas e classificado o estado de hidratação da criança, realizou-se uma comparaçăo ou confronto entre a classificaçāo médica e a "materna", que resultou na constituição de três grupos:

- concordância da classificação "materna" em relação à classificação médica;

- subestimação "materna" do comprometimento do estado de hidratação da criança em relação à classificaçāo fcita pelo médico; e

- superestimação "materna" do comprometimento do estado de hidrataçăo da criança em relação à classificação médica.

Estes grupos foram observados e analisados em função de outras informaçōes:

. instrução materna;

- experiência de desidratação anterior;

- situação nutricional da criança;

- número de dias que a criança levou para chegar ao HMSN;

- se outro serviço de saúde havia sido procurado antes do HMSN por ocasiāo do episódio diarréico.

Ao se colocar a concordância da classificação "matcrna" em relação à classificação médica não se está concebendo esta última como isenta de distorçōes ou falhas, mas simplesmente como suposto referencial científico e técnico para que se pudesse realizar a comparação.

\section{RESULTADOS E DISCUSSÃO}

\section{Características da População em Estudo}

As condiçōes sociais e de vida das crianças 
deste estudo são as de uma população de baixa renda, residentes, na maioria dos casos, no $\mathrm{Mu}-$ nicípio do Rio de Janeiro ( $84 \%$ ) e uma minoria distribuida pela Baixada Fluminense e outros muricípios da Região Metropolitana do Rio de Janeiro (16\%).

Os domicilios encontram-se em áreas de favelas ou loteamentos em $64 \%$ dos casos. Dentre estes domícilios, $85 \%$ são servidos por rede pública de água, $70 \%$ por rede de esgoto, e em $46 \%$ dos mesmos a coleta de lixo é feita pela Prefeitura.

\section{Informações Sobre as Mães e as Crianças}

A maioria das mães é casada ou vive com companheiro $(70,3 \%)$, nāo exercendo atividades profissionais remuneradas fora de casa $(67,6 \%)$. Elas possuem baixo grau de escolaridade, isto é, $80 \%$ das mães têm, no máximo, o primário completo.

Das 406 crianças, $61 \%$ eram do sexo masculino e $39,0 \%$ do sexo feminino. Quanto à idade, $52 \%$ estavam abaixo de 6 meses, $25,6 \%$, entre 6 e 11 meses, e $23,4 \%$, com 12 meses ou mais.

O estado geral dessas crianças no momento do atendimento médico-hospitalar foi considerado regular em $64 \%$ dos casos, como grave em $18 \%$ e bom em outros $18 \%$.

Segundo a classificaçăo médica, estavam desidratadas $70 \%$ das crianças. A desnutrição foi encontrada em 70\% dos casos (classificação Gomez, Padrão NCHS).

Do total das crianças, $72,4 \%$ estavam hospitalizadas, $11,1 \%$ foram atendidas na emergência e receberam reidratação oral no hospital, e $16,5 \%$ receberam alta logo após a consulta médica.

Grupos de Concordância da Classificação "MATERNA" em Relação à Classificação Médica do Estado de Hidrataçăo das Crianças

Pode-se observar, na Tabela 2, alguns sinais e sintomas relacionados ao estado de hidratação das crianças, expostos em níveis de gravidade: 1normal; 2- alterado; 3- muito alterado. Constatase que, dentre eles, alguns são identificados com maior freqüência pelas mães: número de evacuaçðes, número de vômitos, estado de sede, "olhos fundos".

Os sinais e sintomas menos identificados - estado da urina, umidade da boca e da língua, e elasticidade da pele após beliscada - praticamente não têm representação para as mães como indicadores de uma situaçăo de maior gravidade, sendo identificados como normais ou, no máximo, moderadamente alterados.

A identificação mais freqüente do número de evacuaçðes, vômitos, e estado da sede e dos olhos, pode relacionar-se ao fato destas alteraçoes representarem-se por elementos concretos e visíveis que interferem na rotina de cuidado com as crianças e que determinam uma demanda especial da figura materna ${ }^{7}$.

A existência de concordância da classificaçăo dita "materna" em relação àquela realizada pelo médico sobre o estado de hidratação das crianças constitui-se em três grupos: um em que há concordância englobando $35,4 \%$ dos casos, os que a subestimam, $31,3 \%$ e os que a superestimam, $33,3 \%$. Observou-se, portanto, uma distribuição praticamente eqüitativa entre os três grupos.

\section{TABELA 1}

Critérios para classificação do estado de hidratação (recomendado pela OMS)

\begin{tabular}{|c|c|c|c|}
\hline \multirow[t]{2}{*}{ Perguntas } & \multicolumn{3}{|c|}{ Respostas * } \\
\hline & A & B & C \\
\hline Diarréias & 4 dejeçð̋es líquidas/dia & de 4 a 10 dejeçōes líquidas/dia & + 10 dejeções líquidas/dia \\
\hline Vômitos & ausente & pouca quantidade & muito freqüente \\
\hline Sede & normal & aumentada & não pode beber \\
\hline Urina & normal & pouca, escura & nenhuma em $6 \mathrm{~h}$ \\
\hline Olhos & normais & fundos & muito fundos \\
\hline Boca e Língua & molhadas & secas & ressecadas \\
\hline Pele & beliscada, volta ao normal & beliscada, volta lentamente & $\begin{array}{l}\text { beliscada, volta } \\
\text { muito lentamente }\end{array}$ \\
\hline
\end{tabular}

\begin{tabular}{lccc}
\hline Desidratação ? & Se tem estes & Se tem dois ou mais & Se tem dois ou mais destes \\
sintomas & destes sintomas & sintomas \\
Não & Sim, moderada & grave \\
\hline
\end{tabular}

- Os códigos A, B, C correspondem a intensidades crescentes dos sinais e sintomas de desidratação 
TABELA 2

Classificaçăo dos sinais e sintomas de desidratação* identificados pelas mães,

Hospital Salles Neto, Rio de Janeiro, 1987-1988

\begin{tabular}{|c|c|c|c|c|}
\hline $\begin{array}{l}\text { Sinais } \\
e \\
\text { Sintomas }\end{array}$ & Normal & Alterado & $\begin{array}{l}\text { Muito } \\
\text { Alterado }\end{array}$ & Total \\
\hline Evacuaçes & $\begin{array}{l}94 \\
27,9\end{array}$ & $\begin{array}{l}106 \\
31,5\end{array}$ & $\begin{array}{l}137 \\
40,7\end{array}$ & $\begin{array}{l}337 \\
100 \%\end{array}$ \\
\hline Vômitos & $\begin{array}{l}88 \\
27,7\end{array}$ & $\begin{array}{c}153 \\
48,3\end{array}$ & $\begin{array}{l}76 \\
24,0\end{array}$ & $\begin{array}{l}317 \\
100 \%\end{array}$ \\
\hline $\begin{array}{l}\text { Estado de } \\
\text { Sede }\end{array}$ & $\begin{array}{l}100 \\
28,7\end{array}$ & $\begin{array}{l}241 \\
69,3\end{array}$ & $\begin{array}{l}7 \\
2,0\end{array}$ & $\begin{array}{l}348 \\
100 \%\end{array}$ \\
\hline $\begin{array}{l}\text { Estado da } \\
\text { Urina }\end{array}$ & $\begin{array}{l}213 \\
62,3\end{array}$ & $\begin{array}{l}106 \\
31,0\end{array}$ & $\begin{array}{l}23 \\
6,7\end{array}$ & $\begin{array}{l}342 \\
100 \%\end{array}$ \\
\hline $\begin{array}{l}\text { Estado dos } \\
\text { Olhos }\end{array}$ & $\begin{array}{l}72 \\
19,6\end{array}$ & $\begin{array}{l}279 \\
76,0\end{array}$ & $\begin{array}{l}16 \\
4,4\end{array}$ & $\begin{array}{l}367 \\
100 \%\end{array}$ \\
\hline $\begin{array}{l}\text { Estado da } \\
\text { Boca e Língua }\end{array}$ & $\begin{array}{l}122 \\
33,5\end{array}$ & $\begin{array}{l}193 \\
53,0\end{array}$ & $\begin{array}{l}49 \\
13,5\end{array}$ & $\begin{array}{l}364 \\
100 \%\end{array}$ \\
\hline
\end{tabular}

* Segundo critérios da Organizaçăo Mundial da Saúde ${ }^{10}$

TABELA 3

Classificaçăo do Estado de Hidrataçăo* da criança segundo a percepção do médico e da mãe, Hospital Salles Neto, Rio de Janeiro, 1987-1988

\begin{tabular}{|c|c|c|c|c|c|c|c|c|}
\hline \multirow{2}{*}{$\begin{array}{l}\text { Classificaçăo da } \\
\text { Hidrataço Médioo } \\
\text { Classificaçăo } \\
\text { Hidrataçă Mảe }\end{array}$} & \multicolumn{2}{|c|}{$\begin{array}{l}\text { Moderado } \\
\text { Normal }\end{array}$} & \multicolumn{2}{|c|}{ (Desid. I) } & \multicolumn{2}{|c|}{$\begin{array}{c}\text { Grave } \\
\text { (Desid. II e III) }\end{array}$} & \multicolumn{2}{|c|}{ Total } \\
\hline & $\mathbf{N}^{\circ}$ & $\%$ & $\mathbf{N}^{\mathbf{p}}$ & $\%$ & $\mathbf{N}$ & $\%$ & $\mathbf{N}^{\mathbf{2}}$ & $\%$ \\
\hline Normal & $(20)$ & 17,2 & (2) & 2,4 & (2) & 1,5 & (24) & 7,1 \\
\hline Moderado (Desid. D) & (89) & 76,7 & (65) & 78,3 & (101) & 73,7 & (255) & 75,9 \\
\hline Grave (Desid. II e III) & $(7)$ & 6,1 & (16) & 19,3 & (34) & 24,8 & $(57)$ & 17,0 \\
\hline Total & (116) & $100 \%$ & (83) & $100 \%$ & (137) & $100 \%$ & (336) & $100 \%$ \\
\hline
\end{tabular}

OBS: Excluídos os casos de desidrataçăo sem grau especifico

- Critérios da Organização Mundial da Saúde $x^{2}=38.9(p<0,001)$

Pode-se perceber, na Tabela 3, que quando a classificaçăo médica indica um estado de desidrataçăo moderado, é grande a concordância entre esta classificação e aquela que poderia ser realizada pela mãe. Já quando a classificação médica indica normohidrataçăo, a "materna" tende a superestimar o comprometimento do estado de hidratação da criança, classificando-a como desidratada. E quando a classificaçăo médica detecta uma maior gravidade da desidrataçăo das 
crianças, a impressão "materna" tende a subestimar este comprometimento em termos de gravidade.

A evolução da gravidade da desidratação não parece encontrar uma representação para a percepção materna, possivelmente porque esse agravamento seja avaliado através de sinais e sintomas que podem ser menos evidentes ou distorcidos por características que interfiram na sua visualização, tais como alteraç⿸̃es de volume urinário, do turgor e elasticidade da pele ou distinçðes entre "olhos fundos" para "olhos muito fundos" ou "seco" e "ressecado" quanto à umidade da boca e língua.

A percepção dessa passagem de um estado menos grave para um de maior gravidade requer um prévio conhecimento, uma preocupação e atençăo que se voltem para a busca ou deteç̧ăo de sinais e sintomas que o corpo emite e que denunciam a evolução do quadro da desidratação ${ }^{1,15}$.

O conhecimento prévio da importância e dos significados de cada elemento que compőe o quadro de desidratação seria de detecção possível pelas mães se fossem valorizadas as açzes informativas e educativas dentro do cenário de encontro com os profissionais de saúde, seja durante a consulta, na sala de espera, na sala de reidratação oral ou nos horários de visita ${ }^{13}$.

A identificação pelas mães da gravidade da desidratação, ou melhor, dos sinais que permitem esta identificação, é perfeitamente possível e poderia ser de grande benefício tanto para as crianças, quanto para os médicos e para as próprias mães. Isto porque, para as crianças, o esclarecimento da mãe significa o desenvolvimento de atitudes ajustadas no tempo à real forma de apresentação da doença; para os médicos, significa a possibilidade de intervirem precocemente e previnirem situações de maior risco; para as mães, porque isto significa uma maior autonomia e segurança ao lidar com a doença de seus filhos.

A Tabela 4 mostra que as mães que superesti- mariam a classificação médica, isto é, que tenderiam a perceber uma maior gravidade no comprometimento do estado de hidratação de seus filhos, possuem grau de escolaridade um pouco mais elevado em relação aos outros grupos. Aquelas que tenderiam a subestimar a classificaçăo médica, a qual indicaria maior gravidade do que aquela percebida por elas (mães), possuem um nível de escolaridade comparativamente mais baixo.

Embora estas diferenças não sejam estatisticamente significativas, pode-se perceber que ao se comparar faixas extremas do nível de instrução, isto é, mais baixa e mais alta, há um comportamento inverso entre os grupos que superestimariam e subestimariam a desidratação.

A superestimação do comprometimento do estado de hidratação das crianças por um grupo de mães, com melhor nivel de escolaridade, pode estar relacionada a questões como maior acesso a informaçð̃es e maior disponibilidade para apreender e elaborar estas informaçoes e conhecimentos à medida que reconhecem a importância $e$ as razōes que envolvem a sua condiçăo de saúde ${ }^{1}$.

Num outro extremo, tem-se a percepção materna que tenderia a subestimar a classificação médica. A baixa escolaridade desse grupo possivelmente influenciará e determinará formas de apreensão e elaboração dos fatos e das informaç̋̃es recebidas.

Quanto mais baixo for o nível de escolaridade, maiores serão os impedimentos e as dificuldades que se apresentam às mães. As diferenças sociais e culturais entre essas mães e os profissionais de saúde acentuam-se fazendo com que seja mais difícil de se estabelecer um encontro proveitoso ${ }^{6}$.

A desidratação infantil é tema de açóes básicas de saúde onde foram estabelecidos critérios uniformes de diagnóstico e prevenção de quadros mais graves, no sentido da partici pação da população nestas açōes ${ }^{5}$. Contudo, se desconsideradas nesses programas de saúde as diferenças educativas den-

TABELA 4

Concordância entre o médico e a mãe quanto à classificação do estado de hidratação da criança e o nível de instrução materna , Hospital Salles Neto, Rio de Janeiro, 1987-1988

\begin{tabular}{|c|c|c|c|c|c|c|c|c|c|c|}
\hline \multirow{2}{*}{ Concordância } & \multicolumn{2}{|c|}{ Analfabeta } & \multicolumn{2}{|c|}{ Lê/Escreve } & \multicolumn{2}{|c|}{$\begin{array}{c}\text { Instrução da mãe } \\
\text { Primário }\end{array}$} & \multirow{2}{*}{\multicolumn{2}{|c|}{$\underset{N^{2}}{\text { Primeiro grau e }} \underset{\%}{0}+$}} & \multicolumn{2}{|c|}{ Total } \\
\hline & $N^{2}$ & $\%$ & $N^{2}$ & $\%$ & $\mathrm{~N}$ & $\%$ & & & & $\%$ \\
\hline Concorda & 22 & 45,8 & 33 & 42,9 & 58 & 40,8 & 25 & 37,3 & 138 & 41,3 \\
\hline Superestima & 7 & 14,6 & 25 & 32,5 & 50 & 35,2 & 26 & 38,8 & 108 & 32,3 \\
\hline Subestima & 19 & 39,6 & 19 & 24,7 & 34 & 23,9 & 16 & 23,9 & 88 & 26,3 \\
\hline Total & 48 & $100 \%$ & 7 & $100 \%$ & 142 & $100 \%$ & 67 & $100 \%$ & 334 & $100 \%$ \\
\hline
\end{tabular}

$x^{2}=10.19(p>0,05)$ 
tro de um mesmo grupo social, além de diferenças culturais, o bloqueio a esta participação continuará a existir e acabará por reforçar a monopolização do saber e da autoridade de intervenção sobre a problemática de saúde nas mãos de técnicos ou profissionais, que cada vez se posicionam mais distantes das demandas da população ${ }^{2,12}$.

Pode-se observar, na Tabela 5, que a ocorrência de episódios de desidratação anteriores não aumenta a concordância materna em relação ao médico. Isto mostra que o contato com os serviços de saúde, mesmo em episodios de relativa gravidade como a desidratação, deixa um saldo de informação muito pequeno para o paciente ${ }^{5,8}$. Boltanski ${ }^{4}$ reproduz o depoimento de uma mulher acerca do atendimento médico: "Quando dá um medicamento, o médico nunca dá explicacões, dá o remédio e pronto: 'Tome isto e volte depois de amanhã'. Nunca diz porque a gente está doente. Eu gostaria que ele explicasse mais claramente, mas a gente não é mesmo capaz de compreender mais do que isso".

A Tabela 6 revela a situação nutricional das crianças em relação aos grupos de concordância. Constata-se presença mais acentuada de crianças desnutridas nos grupos que subestimariam e concordariam com a classificação médica do estado de hidratação dessas crianças, e, por outro lado, me- nor presença de desnutrição no grupo que superestima a classificação feita pelo médico.

Esta informação pode estar refletindo uma condição de vida dos grupos onde é maior a presença de desnutrição e onde as carências e precariedades sociais e econômicas são intensas e decisivas, acabando inclusive por influenciar na percepção dessas mães ${ }^{6}$, em especial aquelas que subestimariam a gravidade do quadro de desidratação em relação à classificação médica, pois esta subestimação pode representar, entre outras coisas, a desensibilização deste grupo para perceber sua própria situação de vida ${ }^{11,15}$.

Um aspecto importante em relação à presença de desnutrição diz respeito à confluência deste quadro com o de desidratação. Tal combinação pode estar determinando distorçōes e dificuldades na avaliação da real gravidade do quadro de desidratação destas crianças, não só para as mães, mas também para os profissionais de saúde ${ }^{9}$.

Quanto ao tempo decorrido entre o início da diarréia e a chegada ao HMSN (Tabela 7) e o número de serviços procurados antes do referido hospital (Tabela 8), observou-se que mais de $50 \%$ das crianças foram assistidas por pelo menos um serviço de saúde antes de chegarem ao HMSN, por

\section{TABELA 5}

Concordância entre o médico e a mãe quanto à classificação do estado de hidratação da criança segundo a experiência de desidrataçăo anterior, Hospital Salles Neto, Rio de Janeiro, 1987-1988

\begin{tabular}{|c|c|c|c|c|c|c|}
\hline \multirow{2}{*}{ Concordância } & \multicolumn{6}{|c|}{ Desidrataçāo Anterior } \\
\hline & $\mathrm{N}^{\circ}$ & $\%$ & $\mathrm{~N}^{\circ}$ & $\%$ & $\mathrm{~N}^{\circ}$ & $\%$ \\
\hline Concorda & 23 & 34,3 & 109 & 41,8 & 132 & 40,3 \\
\hline Superestima & 26 & 38,8 & 82 & 31,4 & 108 & 32,9 \\
\hline Subestima & 18 & 26,9 & 70 & 26,8 & 88 & 26,8 \\
\hline Total & 67 & $100 \%$ & 261 & $100 \%$ & 328 & $100 \%$ \\
\hline
\end{tabular}

$x^{2}=1.62(p>0,05)$

TABELA 6

Concordância entre o médico e a mãe quanto à classificação do estado de hidratação segundo a situação nutricional da criança, Hospital Salles Neto, Rio de Janeiro, 1987-1988

\begin{tabular}{|c|c|c|c|c|c|c|c|c|}
\hline \multirow{3}{*}{ Concordância } & \multicolumn{8}{|c|}{ Classificação de Gomez } \\
\hline & \multicolumn{2}{|c|}{ Eutrófico } & \multicolumn{2}{|c|}{$\begin{array}{l}\text { Desnutrido } \\
\text { (I) }\end{array}$} & \multicolumn{2}{|c|}{$\begin{array}{l}\text { Desnutrido } \\
\text { (II e III) }\end{array}$} & \multicolumn{2}{|c|}{ Total } \\
\hline & No & $\%$ & $\mathrm{~N}^{\circ}$ & $\%$ & $\mathrm{~N}^{\mathrm{o}}$ & $\%$ & $N^{2}$ & $\%$ \\
\hline Concorda & 35 & 36,5 & 44 & 42,7 & 51 & 45,5 & 130 & 41,8 \\
\hline Superestima & 46 & 47,9 & 19 & 18,4 & 25 & 22,3 & 90 & 28,9 \\
\hline Subestima & 15 & 15,6 & 40 & 38,8 & 36 & 32,2 & 91 & 29,3 \\
\hline Total & 96 & $100 \%$ & 103 & $100 \%$ & 112 & $100 \%$ & 311 & $100 \%$ \\
\hline
\end{tabular}

$x^{2}=38,9(p<0,001)$ 


\section{TABELA 7}

Concordância entre o médico e a mãe quanto à classificaçăo do estado de hidratação da criança segundo o número de dias para chegar ao hospital, Hospital Salles Neto, Rio de Janeiro, 1987-1988

\begin{tabular}{|c|c|c|c|c|c|c|}
\hline \multirow{3}{*}{ Conoordância } & \multicolumn{6}{|c|}{ Número de Dias } \\
\hline & \multicolumn{2}{|c|}{1 a 3 dias } & \multicolumn{2}{|c|}{$4 e+$ dias } & \multicolumn{2}{|c|}{ Total } \\
\hline & $\mathbf{N}^{0}$ & $\%$ & $\mathbf{N}$ & $\%$ & $\mathbf{N}^{\mathbf{p}}$ & $\%$ \\
\hline Concorda & 53 & 36,6 & 80 & 43,2 & 133 & 40,3 \\
\hline Superestima & 57 & 39,3 & 51 & 27,6 & 108 & 32,7 \\
\hline Subestima & 35 & 24,1 & 54 & 29,2 & 89 & 27,0 \\
\hline Total & 145 & $100 \%$ & 185 & $100 \%$ & 330 & $100 \%$ \\
\hline
\end{tabular}

$x^{2}=5.10(p>0,05)$

TABELA 8

Concordância entre o médico e a mãe quanto à classificação do estado de hidrataçăo segundo os serviços de saúde procurados por ocasião do episódio diarréico, antes de chegar ao Hospital Salles Neto, Hospital Salles Neto, Rio de Janeiro, 1987-1988

\begin{tabular}{|c|c|c|c|c|c|c|c|c|}
\hline \multirow[b]{2}{*}{ Concordância } & \multicolumn{8}{|c|}{ Serviços Procurados } \\
\hline & \multicolumn{2}{|c|}{ Nenhum } & \multicolumn{2}{|c|}{$\begin{array}{l}1-2 \text { Serviços } \\
\mathbf{N}^{\mathbf{2}} \%\end{array}$} & \multicolumn{2}{|c|}{$\begin{array}{c}3 \underset{\mathrm{e}}{\mathrm{e}}+\text { Serviços } \\
\mathrm{N}^{2}\end{array}$} & \multicolumn{2}{|c|}{ Total } \\
\hline Concorda & 45 & 32,4 & 74 & 49,0 & 14 & 37,8 & 133 & 40,7 \\
\hline Superestima & 68 & 48,9 & 30 & 19,9 & 8 & 21,6 & 106 & 32,4 \\
\hline Subestima & 26 & 18,7 & 47 & 31,1 & 15 & 40,5 & 88 & 26,9 \\
\hline Total & 139 & $100 \%$ & 151 & $100 \%$ & 37 & $100 \%$ & 327 & $100 \%$ \\
\hline
\end{tabular}

$x^{2}=32.38(p<0,001)$

ocasiåo deste epiśdio diarréico. $O$ grupo de mães que superestimaria a classificação médica chegou mais rápido ao HMSN, menos de três dias após o início da diarréia na maioria dos casos.

Por outro lado, em mais da metade dos casos, o grupo que subestimaria a classificação médica levou quatro dias ou mais para chegar ao mesmo hospital. Esta informação pode estar vinculada ao que se observa na Tabela 8, onde a peregrinação de mães e crianças deste grupo pelos serviços de saúde é maior em relação aos outros dois.

Embora no Município do Rio de Janeiro seja alta a cobertura de serviços, particularmente dos serviços públicos, acredita-se que a peregrinação de mães e crianças esteja relacionada a fatos tais como a baixa resolutividade e qualidade desses serviços, bem como acessibilidade (repressão de demanda) aos serviços disponíveis e conhecimento dos mesmos pela população.

A percepção materna da gravidade do comprometimento do estado de hidratação das crianças pode estar influenciando a busca por uma assistência médico-hospitalar devido ao retardo ou imediatismo em obter esta assistência ${ }^{14}$. Pode-se levantar algumas possibilidades que envolveriam esta procura pela assistência aos problemas de saúde, ou seja, a percepção de que o estado das crianças não necessitasse dessa assistência, ou o fato deste tipo de assistência atualmente prestado ser de tão difícil acesso, talvez tenha acabado por gerar uma atitude de descrédito e imobilização por parte dessas mães, que retardariam ao máximo a procura por um serviço de saúde.

Segundo Berlinguer ${ }^{3}$, o sentir-se doente está ligado com freqüência à percepçăo do proprio corpo. Nas pessoas que pertencem as classes populares, é constantemente escassa a cultura somática (do grego somo "corpo"), enquanto que nas classes abastadas é mais comum se ouvir os sinais que vêm do próprio corpo 4 . A doença acontece freqüentemente de improviso e de forma mais grave nos primeiros, uma vez que não é enfrentada a tempo.

Concluindo, depreende-se que às mães é perfeitamente possivel identificar a desidratação e sua gravidade pelos sinais e sintomas, à medida em que elas conheçam os significados dessas alteraçóes. Esse esclarecimento deve ser promovido principalmente pelos profissionais de saúde, que são os que fazem a intermediação entre os conhecimentos que possuem e a realidade de saúde e de vida da população, permitindo, desta forma, ampliar a consciência social desses grupos, freqüentemente encarados como incapazes nesta relação. 
LEAL, M. C. et al. [Maternal perception of dehydration in children with diarrhoea. A study of agreement with medical diagnosis]. Rev. Saúde puibl. S. Paulo, 24: 196-203, 1990.

ABSTRACT: The mother's perception of signs and symptoms of dehydration in children under three years of age was studied and compared with the medical classification. The study was carried out in a children's hospital in the city of Rio de Janeiro, among children hospitalized with diarrhoea between January, 1987 and February, 1988. The number of excretions and of vomitings, thirst and condition of eyes constituted the signs and symptoms most frequently reported by mothers. However, they had difficulty in judging the amount of urine, humidity of mouth and tongue and turgidity of the skin. These signs were almost always regarded as normal or, at most, as indicating only slight alteration. Those mothers who tended to underestimate the severity of the dehydration indicated by the physician were of a lower educational level and had more severely undernourished children and greater difficulty of access to the hospital. On the other hand, those who tended to overestimate it belonged to a higher educational level, had better-nourished children, greater ease of access to the hospital and were attended to by a smaller number of health care services before reaching the hospital surveyed. Those who agreed with the medical diagnosis were in an intermediate situation, although they tended to be closer to those who underestimated the gravity of the dehydration. Those mothers whose children had already gone through a dehydration episode did not present a more intense agreement with the physician's diagnosis, thus evidencing that the information afforded at the health care service was either non-existent or inadequate.

KEYWORDS: Mothers. Perception. Dehydration, epidemiology.

\section{REFERENCIAS BIBLIOGRÁFICAS}

1. ANGATE-YANGNI, A. Understanding traditional medicine. Wld Hlih Forum, 2: 240-4, 1981.

2. BARBOSA, F. S. O. As utopias da educação médica. Rev. bres. Educ. méd., 7 (1): 7-8, 1983.

3. BERLINGUER, G. A doenga, Såo Paulo, Hucitec, 1988.

4. BOLTANSKI, L. As classes sociais e o corpo. Rio de Janeiro, Graal, 1984.

5. EISEMON, T. O.; PATEL, V. L R.; SENZ, S. O. Uses of formal and informal knowledge in the comprehension of instructions for oral rehidratation therapy in Kenya. Soc. Sci. Med., 25: 1225-34, 1987.

6. FONAROFF, A. Percepciones culturales y transtomos nutricionales: estudio de casos de Jamaica. Bol. Ofic. sanit. panamer., 80: 135-50, 1976.

7. FORYSTH, B. W. C.; LEVENTHAL, J. M.; Mc CARTHY, P. L. Mother's perceptions of problems of feeding and crying behaviors. Amer. J. Dis. Child., 139: 269-72, 1985.

8. MALISON, M. D.; SEKEITO, P.; HENDERSON, P. L.; HAWKINS, R. V.; OKWARE, S. I.; JONES, T. S. Estimating health service utilization, immunization coverage and childhood mortality: new approach in Uganda. Bull. Wld Hith Org., 65: 265 424, 1987.9. MARCONDES, E. Pediatria básica. $7 \mathfrak{c d}$ ed Săo Paulo, Savier, 1986.
10. ORGANZZAÇÃO MUNDIAL DA SAÚDE. Programa de Lucha contra las Enfermedades Diarreicas. Manual para el tratamiento de la diarrea aguda. Ginebra, s.d.

11. PELTO, G. H. Cultural issues in matemal and child health and nutrition. Soc. Sci. Med., 25: 553-9, 1987.

12. SANTOS, J. O. Contribuição ao estudo dos valores, finalidades e objetivos da educaçăo médica. Rev. bras. Educ. Méd., 11: 1-36, 1987.

13. SPARR, L. F.; GORDON, G. H.; HICKAM, D. H.; GIRARD, D. H. The doctor patient relationship during medical intership: the evolution of dissatisfaction. Soc. Sci. Med., 26: 1095-101, 1988.

14. TSUI, A. O.; DECLERQUE, J.; MANGANI, N. Matemal and sociodemographic correlates of child morbidity in Bas Zaire: the effects of matemal reporting. Soc. Sci. Med., 26: 701-13, 1988.

15. TUCKER, K. \& SANJUR, D. Maternal employment and child nutrition in Panama. Soc. Sci. Med., 26: $605 \cdot 12,1988$

Recebido para publicaçáo em 20109/1989 Aprovado para publicaçẽo em 23101/1990 